\title{
Low concentration flufenamic acid enhances osteogenic differentiation of mesenchymal stem cells and suppresses bone loss by inhibition of the NF-KB signaling pathway
}

\author{
Xuenan Liu', Zheng Li', Hao Liu', Yuan Zhu', Dandan Xia², Siyi Wang ${ }^{1}$, Ranli Gu', Weiliang Wu ${ }^{3}$, Ping Zhang ${ }^{1 *}$, \\ Yunsong Liu' ${ }^{1 *}$ and Yongsheng Zhou ${ }^{1}$
}

\begin{abstract}
Background: As the representative of fenamic acids, an important group of NSAIDs, flufenamic acid (FFA) has been used for anti-inflammation and analgesia in the clinic. Recently, researches have focused on the role of some members of NSAIDs in promoting osteogenesis. However, little attention has been paid to the subgroup of fenamic acids, and it remains unclear whether FFA and other fenamic acids could regulate mesenchymal stem cells' (MSCs) lineage commitment and bone regeneration.

Methods: Here we treated two kinds of human MSCs with FFA at different concentrations in vitro and examined the effect of FFA on osteogenic differentiation of human MSCs. This was followed by heterotopic bone formation assay in nude mice. In addition, ovariectomized and aged mice were used as osteoporotic models to test the effect of FFA on osteoporosis. Besides, activators and inhibitor of nuclear factor-KB (NF-kB) signaling pathway and western blot were used to clarify the mechanism of the promoting effect of low concentration FFA on osteogenesis.

Results: Our results indicated that low concentrations of FFA could significantly enhance osteogenic differentiation of human MSCs in vitro, as well as in vivo. In addition, FFA treatment suppressed bone loss in ovariectomized and aged mice. Mechanistically, FFA at low concentrations promoted osteogenesis differentiation of human MSCs by inhibition of the NF-kB signaling pathway.
\end{abstract}

Conclusions: Collectively, our study suggested that low concentration FFA could be used in bone tissue engineering or osteoporosis by promoting osteogenic differentiation of human MSCS.

Keywords: Flufenamic acid, Mesenchymal stem cells, Osteogenesis, Osteoporosis, Nuclear factor-KB

\section{Background}

As a common disease that can often be seen in the clinic, osteoporosis brings health and economic burdens to patients and remains a clinical challenge. It is usually caused by estrogen deficiency in menopausal women or by aging

\footnotetext{
* Correspondence: zhangping332@bjmu.edu.cn; liuyunsong@hsc.pku.edu.cn ${ }^{1}$ Department of Prosthodontics, Peking University School and Hospital of Stomatology, National Laboratory for Digital and Material Technology of Stomatology, Beijing Key Laboratory of Digital Stomatology, National Clinical Research Center for Oral Diseases, 22 Zhongguancun South Avenue, Beijing 100081, People's Republic of China

Full list of author information is available at the end of the article
}

in men [1], leading to low bone strength, caused by low mineral density, architectural deterioration of the bone structure, and susceptibility to fracture [2]. Previous studies have shown that abnormal in osteogenic differentiation capacity of bone marrow-derived mesenchymal stem cells (BMMSCs) contributes to these structural abnormalities [3-5], which is mainly caused by loss of bone homeostasis.

Non-steroidal anti-inflammatory drugs (NSAIDs) are commonly used in the clinic and can inhibit the synthesis of prostaglandin by inhibiting cyclooxygenases (COXs) to achieve the anti-inflammatory and analgesia

(c) The Author(s). 2019 Open Access This article is distributed under the terms of the Creative Commons Attribution 4.0 International License (http://creativecommons.org/licenses/by/4.0/), which permits unrestricted use, distribution, and 
effects [6-8]. However, the members of the NSAIDs are quite different in their chemical structures and other properties, which may cause different effects in addition to anti-inflammatory and analgesia effects. According to their chemical structure, NSAIDs can be divided into several groups, for instance, salicylic acids, anilines, acetic acids, oxicams, and fenamic acids [9]. Some members of NSAIDs have been proven to have complex effects on the biological behaviors of mesenchymal stem cells (MSCs) and bone homoeostasis, such as aspirin [10], ibuprofen, and indomethacin [11]. However, little attention has been paid to the important group of fenamic acids. In particular, the effect of fenamic acids on MSC fate has not been reported.

A variety of transcription and signaling factors participate in lineage commitment of MSCs toward osteogenic cells, including the nuclear factor kappa B (NF- $\mathrm{kB}$ ) signaling pathway. As a regulator of immune and inflammatory signals $[12,13]$, the NF-kB signaling pathway plays an important role in regulating the differentiation and function of osteocytes and in bone metabolism [14-18]. Activation of this pathway can prevent osteogenic differentiation of MSCs [14] and inhibit osteoblastic SMAD activation [18] and the synthesis of matrix protein in bone [19], as well as suppressing postnatal bone formation in vivo [15]. Besides, several genes participate in bone metabolism through the NF- $\mathrm{KB}$ signaling pathway [20, 21]. However, whether fenamic acids can affect the functions of MSCs through the NF- $\mathrm{kB}$ signaling pathway remains unknown.

In the present study, we aimed to explore the in vitro and in vivo effects of FFA, a representative of the fenamic acids of NSAIDs, on the osteogenic differentiation of hMSCs and its potential mechanism. The in vivo effects of FFA in an osteoporotic animal model were also involved. Our results demonstrated that low concentration FFA promoted osteogenic differentiation of hMSCs in vitro and the effect declined with the concentration increasing. The best concentration for osteogenesis promoting was $50 \mu \mathrm{M}$. Fifty micromolar FFA could also promote osteogenic differentiation of hMSCs in vivo. Besides, $50 \mu \mathrm{M}$ FFA suppressed bone loss in OVX mice and aged mice. Mechanistically, FFA promoted osteogenesis through inhibition of NF- $\mathrm{kB}$ signaling pathway. Overall, our study provided valuable clues for the potential use of FFA to treat bone metabolic disease and in bone tissue engineering.

\section{Methods}

\section{Culture and osteogenic induction of hMSCs}

Primary human BMMSCs and human adipose-derived mesenchymal stem cells (hASCs) were purchased from ScienCell Company (San Diego, CA, USA). All cellbased in vitro studies were repeated three times using MSCs from three donors. All materials were purchased from Sigma-Aldrich (St. Louis, MO, USA) unless otherwise stated. This study was approved by the Institutional Animal Care and Use Committee of the Peking University Health Science Center (LA2014233), and all experiments were performed in accordance with the approved guidelines.

FBS, MEM, DMEM, and 100× penicillin and streptomycin mixture were purchased from Gibco (Grand Island, NY, USA). Human BMMSCs and ASCs were cultured in proliferation medium (PM), consisting of $10 \%(v / v)$ FBS, penicillin/streptomycin, and fresh MEM (for hBMMSCs) or DMEM (for hASCs), with $5 \% \mathrm{CO}_{2}$ atmosphere at $37^{\circ} \mathrm{C}$. The OM comprised fresh DMEM or MEM containing $10 \mathrm{nM}$ dexamethasone, $10 \mathrm{mM} \beta$ glycerophosphate, $0.2 \mathrm{mM}$ L-ascorbic acid, $10 \%(v / v)$ FBS, and penicillin/streptomycin. TNF- $\alpha$ was purchased from R\&D Systems (Minneapolis, MN, USA), and BAY117082 was purchased from Selleck (Houston, TX, USA). Cells at the fourth to sixth passage were used for the in vitro experiments.

\section{Preparation of concentrated FFA solution}

Flufenamic acid was first dissolved in DMSO to obtain a concentrated solution at $200 \mathrm{mmol} \mathrm{L}^{-1}$, to test the effect of FFA at different concentrations on the differentiation of hMSCs, and to choose the optimal concentration for osteogenic differentiation of hMSCs in vitro.

\section{Cell proliferation assay}

Human BMMSCs or ASCs were seeded in 12-well plates at $2 \times 10^{4}$ per well. After that, the cells were cultured in PM, or PM with $25,50,100$, and $200 \mu \mathrm{M}$ FFA, respectively. Three wells of cells in the same treatment method were tested daily from day 1 to day 7 . The cells were counted using a Cell Counting Kit-8 (CCK8, Dojindo Laboratories, Kumamoto, Japan), according to the manufacturer's instructions and growth curves of the cells were obtained according to the cell number.

\section{Alkaline phosphatase staining}

Human BMMSCs or ASCs were seeded in 6-well plates at the same initial density. After 7 days of culture in PM, $\mathrm{OM}$, or $\mathrm{OM}$ with different concentrations of FFA, the cells were washed with PBS, fixed in 95\% cold ethanol, and washed with PBS again. An alkaline phosphatase (ALP) Staining Kit (CWBIO, Beijing, China) was used for staining, according to the manufacturer's instructions. The cells were then gently washed with distilled water, and images were obtained using a scanner.

\section{Quantification of ALP activity}

Human BMMSCs or ASCs were seeded in 6-well plates at the same initial density. After 7 days of culture in PM, $\mathrm{OM}$, or $\mathrm{OM}$ with different concentrations of FFA, the 
cells were gently washed with PBS first. Then, the cells were collected and centrifuged at $13362 \times g$ for $30 \mathrm{~min}$ at $4{ }^{\circ} \mathrm{C}$ after being lysed with $1 \%$ Triton X-100 on ice. A BCA protein assay kit (Pierce Thermo Scientific, Waltham, MA, USA) was used to measure the total protein concentration, according to the manufacturer's instructions. An ALP assay kit (Nanjing Jiancheng Bioengineering Institute, Nanjing, China) was used to measure the ALP activity, and the ALP activity was normalized to the total protein content of each sample.

\section{Alizarin red $\mathrm{S}$ staining and quantification}

Human BMMSCs or ASCs were seeded in 6-well plates at the same initial density. After 14 days (hBMMSCs) or 21 days (hASCs) of culture in PM, OM, or OM with different concentrations of FFA, cells were gently washed with PBS, fixed with 95\% cold ethanol, and washed with distilled water. Two percent alizarin red S (ARS) staining solution was then used to stain the cells for $20 \mathrm{~min}$ at room temperature. After that, the images were scanned.

For alizarin red S quantification, the stained cells were solubilized with $100 \mathrm{mM}$ cetylpyridinium chloride and the OD value of the solution in each well at $562 \mathrm{~nm}$ was then measured spectrophotometrically.

\section{Quantitative real-time reverse transcription PCR}

Total RNA was extracted using the TRIzol reagent (Invitrogen, Carlsbad, CA, USA) from hBMMSCs or hASCs cultured in different media for 7 or 14 days, and a Nano Drop 8000 spectrophotometer (Pierce Thermo Scientific) was used to determine the purity and concentration of the total RNA. A PrimeScript RT Reagent Kit (Takara, Tokyo, Japan) was used to perform reverse transcription, according to the manufacturer's instructions. The mRNA expression of each gene was then tested by qPCR using the SYBR Green Master Mix (Roche Applied Science, Mannheim, Germany) and a 7500 Real-Time PCR Detection System (Applied Biosystems, Foster City, CA, USA) with glyceraldehyde-3-phosphate dehydrogenase (GAPDH) as the reference gene. The primer sequences of human GAPDH, ALP, RUNX2, BGLAP, TNF, IL6, IL8, and ICAM1 used for real-time PCR were as follows: GAPDH, (forward) 5'-GAAGGTGAAGGTCGGAGTC-3' and (reverse) 5'-GAAGATGGTGA TGGGATTTC-3'; $A L P$, (forward) 5' -ATGGGATGGGTGTCTCCACA-3' and (reverse) 5'-CCACGAAGGGGAACTTGTC-3'; RUNX2, (forward) 5'-CCGCCTCA GTGATTTAGGGC-3' and (reverse) 5'-GGGTCTGTAATCTGACTCTGTCC-3'; BGLAP, (forward) 5'-CACTCCTCGCCCTATTGGC-3' and (reverse) 5'-CCCTCCT GCTTGGACACAAAG-3'; TNF, (forward) 5'-GCCCAGGCAGTCAGATCATCTT C-3' and (reverse) 5'-ACAGGCTTGTCACTCGGGGT -3'; IL6, (forward) 5'-GCCAG AGCTGTGCAGATGA GT-3' and (reverse) 5' -AGCA GGCTGGCATTTG
TGGT-3'; IL8, (forward) 5' -ACCACACTGCGCCAACA CAG-3' and (reverse) 5'-TGCACCCA GTTTTCCT TGGGG-3'; and ICAM1, (forward) 5'-TGCACCCAGT TTTCCTTGGG G-3' and (reverse) 5'-GGCGCCGG AAAGCTGTAGAT-3'.

\section{Western blot}

First, hBMMSCs were washed with cold PBS softly for three times. After that, the cells were lysed in radioimmunoprecipitation assay (RIPA) buffer supplemented with $1 \%$ phosphatase inhibitor (Roche) and $2 \%$ protease inhibitor cocktail (Roche). After collecting and centrifugation of the cell lysate at $13362 \mathrm{~g}$ at $4{ }^{\circ} \mathrm{C}$ for $30 \mathrm{~min}$, supernatants were carefully transferred to new tubes, and the $\mathrm{BCA}$ protein assay kit was used to determine the protein concentrations. Thirty-five-microgram total protein of each sample was subjected to $10 \%$ SDS-PAGE. Proteins were transferred to a polyvinylidene fluoride membrane (Millipore, Billerica, MA, USA) after electrophoresis. The membrane was then blocked with $5 \%$ nonfat milk and then incubated with anti-IkB $\alpha$ (Abcam, Cambridge, UK), anti-p-IKK, anti-p-IKB $\alpha$, anti-p-P65, anti-P65, anti-RUNX2, or anti-GAPDH (Cell Signaling Technology, Beverly, MA, USA) in TBS at $4{ }^{\circ} \mathrm{C}$ overnight. After that, the membrane was washed with Trisbuffered saline-Tween 20 (TBST) buffer for 3 times and then incubated with goat anti-rabbit IgG (Abcam). After that, the membrane was washed with TBST buffer again. Lastly, an ECL Western blot kit (CWBIO) was used to visualize the bands.

\section{FFA treatment of transplants in vivo}

All the mice used in the present study were purchased from Vital River Corporation (Beijing, China) and were maintained in a pathogen-free facility on a 12-h light/ dark cycle with water and food provided ad libitum.

For heterotopic bone formation, 6-week-old $(n=18)$ female BALB/C homozygous nude mice were randomly divided into three groups (6 mice per group): PM, $\mathrm{PM}+\mathrm{DMSO}$, and PM+50 $\mu \mathrm{M}$ FFA. Next, hBMMSCs for the three groups were cultured in PM, PM with $1 \%$ o DMSO, and PM with $50 \mu \mathrm{M}$ FFA (FFA was first dissolved in DMSO to get a concentrated solution at 50 $\mathrm{mmol} \mathrm{L}{ }^{-1}$ ), respectively, for 10 days. After that, the cells in each treatment group were harvested and incubated with tricalcium phosphate (TCP) carrier (Bicon, Boston, MA, USA) scaffolds at $37^{\circ} \mathrm{C}$ for $1 \mathrm{~h}$, followed by centrifugation at $150 \times g$ for $5 \mathrm{~min}$. The hBMMSCs-scaffolds hybrids were implanted into the dorsal subcutaneous space of the nude mice. The samples were carefully harvested after 8 weeks and analyzed by H\&E and Masson staining. 


\section{Ovariectomy and sham operations}

Female C57BL6 mice (8 weeks old $(n=40))$ were randomly divided into two groups. For general anesthesia, pentobarbital sodium $\left(50 \mathrm{mg} \mathrm{kg}^{-1}\right)$ was given to the mice by intraperitoneal injections. After that, a bilateral ovariectomy (OVX) or sham operation was performed using standard methods [22].

\section{In vivo experiment with FFA injection}

Four weeks after surgery [23], the 40 OVX or sham mice were randomly divided into four groups of 10 mice for FFA or normal saline (N.S.) injections as follows: (1) Sham mice with N.S.; (2) Sham mice with FFA; (3) OVX mice with N.S.; and (4) OVX with FFA. The in vivo treatment dose was calculated according to the best concentration of promoting osteogenesis in vitro, $50 \mu \mathrm{M}$ as follows:

An adult mouse $(\sim 25 \mathrm{~g}$ in weight) has a circulating blood volume of $\sim 2.5 \mathrm{~mL}$ (http://web.jhu.edu/animal care/procedures/mouse.html). The blood concentration of FFA at $50 \mu \mathrm{M}$, the optimal concentration for the in vitro study, was used, and the injection amount of FFA should be $\sim 1.4 \mathrm{mg} \mathrm{kg}^{-1}$ (the molecular weight of FFA is $281.23 \mathrm{~g} \mathrm{~mol}^{-1}$ ) [24].

After 1 month of injection of FFA or N.S. with the same volume as the FFA solution per day, the mice were sacrificed and their femurs, hearts, livers, spleens, lungs, and kidneys were carefully collected and fixed in 10\% formalin. The blood of each mouse was also carefully collected.

For aged mice, 12-month-old female C57BL6 mice $(n=20)$ were randomly divided into two groups of 10 mice for FFA or N.S. injections. Mice in group one (FFA) were given FFA at $1.4 \mathrm{mg} \mathrm{kg}^{-1}$ per day through intraperitoneal injection. Mice in group two (N.S.) were given N.S. in the same volume as the FFA solution. One month later, the mice were sacrificed, and their femurs, hearts, livers, spleens, lungs, and kidneys were carefully collected and fixed in 10\% formalin. The blood of each mouse was also carefully collected.

\section{Analysis of serum biomarker}

Serum procollagen I N-terminal propeptide (P1NP) was chosen as the indicator of bone formation. The serum biomarker was measured using an ELISA kit (CloudClone, Katy, TX, USA) according to the manufacturer's instructions.

\section{Bone histomorphometry}

Bone histomorphometry analysis was performed following a protocol that has been described [25]. To be specific, calcein was given to the mice through intraperitoneal injection at $10 \mathrm{mg} \mathrm{kg}^{-1} 8$ days before they were euthanized for histomorphometry analysis. Seven days after the first injection, calcein was injected again at the same dose. The femurs were carefully collected and fixed in $10 \%$ formalin. The bones were then dehydrated, embedded with resin, and sliced for H\&E staining. All bone-specific parameters were then measured and calculated.

\section{Micro-computed tomography and bone morphometric analysis of mice}

The femurs were carefully collected and then fixed with $10 \%$ formalin for $24 \mathrm{~h}$ before being gently washed with $10 \%$ sucrose solution. After that, micro-CT images were obtained at a resolution of $8.82 \mu \mathrm{m}$, with a tube voltage of 60 $\mathrm{kV}$, a tube current of $500 \mu \mathrm{A}$, and an exposure time of 1500 ms. Multimodal 3D visualization software (Inveon Research Workplace; Siemens, Munich, Germany) supplied by the micro-CT system was used for three-dimensional (3D) reconstructions on the basis of two-dimensional (2D) images.

An Inveon Research Workplace (Siemens) was used to calculate the parameters: Bone mineral density (BMD), bone volume/total volume (BV/TV), trabecular thickness (Tb.Th), trabecular number (Tb.N), and trabecular separation (Tb.Sp) in the region of interest $(0.5$ to $1 \mathrm{~mm}$ distal to the proximal epiphysis).

\section{Statistical analysis}

All statistical analyses were performed using SPSS Statistics 20.0 software (IBM). Independent two-tailed Student's $t$ tests were used to analyze the comparisons between two groups. One-way ANOVA and a Tukey's post hoc test were used to analyze the comparisons between more than two groups. Data shown represents the mean \pm standard deviation (SD) of 3 to 10 experiments per group. Values of $p<0.05$ were considered statistically significant.

\section{Results}

FFA at low concentrations promoted osteogenic differentiation of hBMMSCs in vitro

ALP staining and quantification showed that low concentration FFA promoted the osteogenic differentiation of hBMMSCs cultured in OM on day 7 (Fig. 1a, c). The ARS staining and quantification on day 14 displayed similar results to those of ALP staining and quantification (Fig. 1b, d). FFA at 25, 50, and $100 \mu \mathrm{M}$ increased the expression level of $A L P, R U N X 2$ (Fig. 1e) at day 7, and BGLAP (Fig. 1f) at day 14. However, the promoting effect declined with increasing concentration. The best concentration for osteogenesis promoting was $50 \mu \mathrm{M}$. When the concentration reached $200 \mu \mathrm{M}$, FFA inhibited osteogenic differentiation (Fig. 1a-f). In addition, the presence of 1\%o DMSO did not have any influence on the osteogenic differentiation of hBMMSCs in vitro (Additional file 1: Figure S1a). Furthermore, 25 to $100 \mu \mathrm{M}$ FFA did not affect the proliferation of hBMMSCs in vitro, whereas $200 \mu \mathrm{M}$ FFA 


\section{hBMMSCs}

\section{PM}

$\mathbf{a}$

$\mathbf{b}$

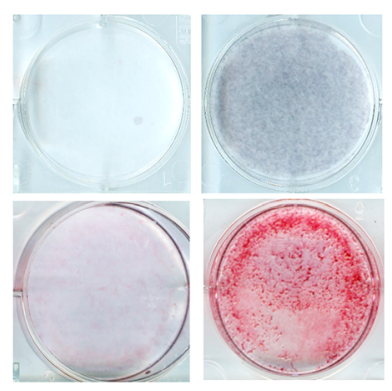

c

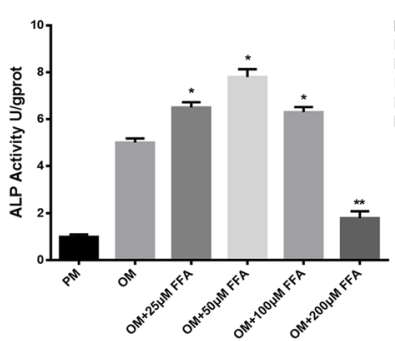

e

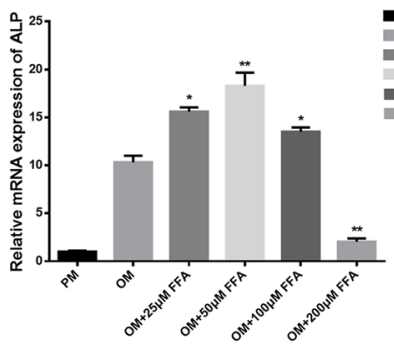

f

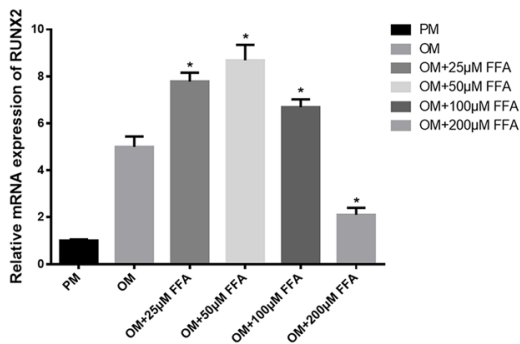

OM+FFA

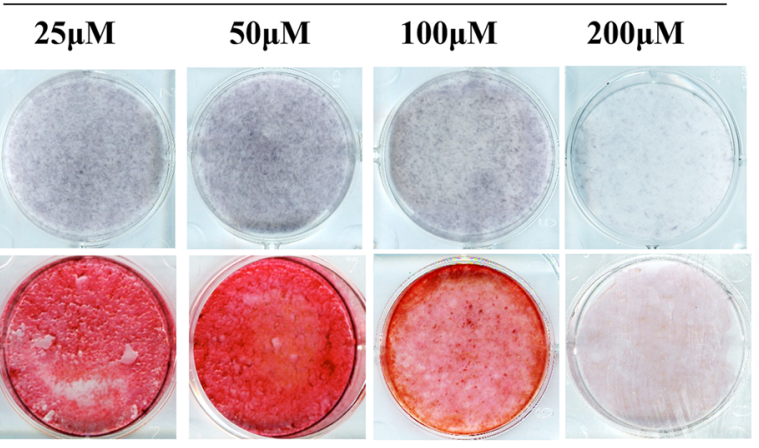

d

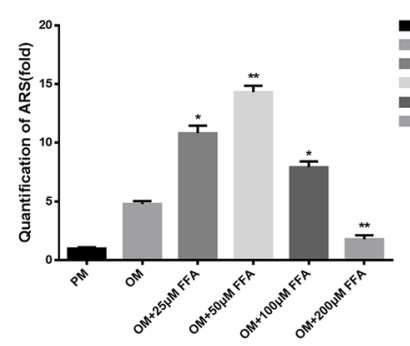

- PM

OM+25IMFFA

OM+50MMFA

$O M+100 \mathrm{MMFA}$
$\mathrm{OM}+200 \mathrm{MM} \mathrm{MFA}$

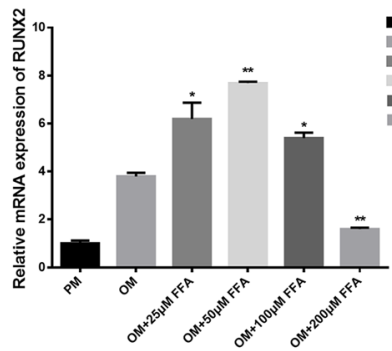

- $\mathrm{PM}$

OM+25uM FFA

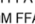

OM+200MM FFA

on

OM+255MFFA

- OM+100uMFFA

OM+200uM FFA

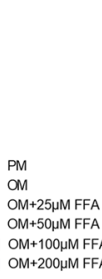

\section{(1)}

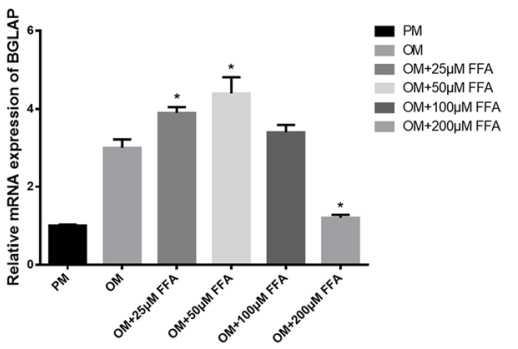

Fig. 1 FFA at different concentrations affected osteogenic differentiation of hBMMSCs in vitro. a 25, 50, and $100 \mu M$ FFA increased ALP activity in hBMMSCs, whereas 200 MM FFA decreased ALP activity as shown by ALP staining. Human BMMSCs were cultured in PM, OM, or OM with FFA at 25, 50,100, or $200 \mu \mathrm{M}$ for 7 days. b FFA at 25, 50, and $100 \mu \mathrm{M}$ accelerated mineralization in hBMMSCs, whereas $200 \mu \mathrm{M}$ FFA reduced mineralization as shown by ARS staining. Human BMMSCs were cultured in PM, OM, or OM with FFA at 25, 50,100, or $200 \mu \mathrm{M}$ for 14 days. c The result of quantification of ALP activity was consistent with the result of ALP staining. $\mathbf{d}$ The result of quantification of ARS was consistent with the result of ARS staining. e FFA at 25, 50, and $100 \mu \mathrm{M}$ promoted the expression of ALP and RUNX2 on day 7 . However, $200 \mu \mathrm{M}$ FFA inhibited the expression of the genes mentioned above, as determined by qRT-PCR. f FFA at 25, 50, and $100 \mu \mathrm{M}$ promoted the expression of RUNX2 and BGLAP on day 14 , whereas $200 \mu \mathrm{M}$ FFA inhibited the expression of the genes mentioned above, as determined by qRT-PCR in hBMMSCs. All data are shown as the mean $\pm S D, n=3 .{ }^{*} p<0.05$ and ${ }^{* *} p<0.01$ compared with OM. FFA, flufenamic acid; hBMMSC, human bone marrow-derived mesenchymal stem cell; PM, proliferation media; OM, osteogenic media; ALP, alkaline phosphatase; ARS, alizarin red S; RUNX2, runt-related transcription factor 2; $B G L A P$, bone gamma-carboxyglutamate protein; GRT-PCR, quantitative real-time reverse transcription PCR 
inhibited the proliferation of hBMMSCs in vitro (Additional file 2: Figure S2a).

\section{FFA at low concentrations promoted osteogenic differentiation of hASCs in vitro}

Similar results were obtained in another kind of hMSCs, hASCs. ALP staining and quantification showed that low concentration FFA promoted the osteogenic differentiation of hASCs cultured in OM on day 7 (Fig. 2a, c). The ARS staining and quantification on day 14 displayed similar results to those of ALP staining and quantification (Fig. 2b, d). FFA at 25, 50, and $100 \mu \mathrm{M}$ increased the expression level of $A L P, R U N X 2$ (Fig. 2e) at day 7 , and BGLAP (Fig. 2f) at day 14. However, the promoting effect declined with increasing concentration. The best concentration for osteogenesis promoting was $50 \mu \mathrm{M}$. When the concentration reached $200 \mu \mathrm{M}$, FFA inhibited osteogenic differentiation (Fig. 2a-f). In addition, the presence of $1 \%$ DMSO did not have any influence on the osteogenic differentiation of hASCs in vitro (Additional file 1: Figure S1b). Furthermore, 25 to $100 \mu \mathrm{M}$ FFA did not affect the proliferation of hASCs in vitro, whereas $200 \mu \mathrm{M}$ FFA inhibited the proliferation of hASCs in vitro. (Additional file 2: Figure S2b).

\section{Low concentration FFA enhanced osteogenic differentiation of hBMMSCs in vivo}

According to the in vitro experiments, $50 \mu \mathrm{M}$ FFA had the best promoting effect on osteogenesis. So, $50 \mu \mathrm{M}$ FFA was used in the following in vivo experiments. Human [BMMSCs] were treated in the following three ways: PM, PM with $1 \% \mathrm{DMSO}$, and PM with $50 \mu \mathrm{M}$ FFA for 10 days. (The treated cells) were loaded onto TCP carrier scaffolds and implanted in the dorsal subcutaneous space of nude mice (six mice per group). We collected the implantation samples after 8 weeks and performed the analysis. H\&E staining revealed more newly formed bone in the PM+FFA group than in the other two groups (Fig. 3a). Masson's trichrome staining also showed that there was more collagen organization (blue color) in the PM+FFA group than in the other two groups (Fig. 3b). There were no significant differences between the PM group and PM+DMSO group, which means the presence of $1 \%$ DMSO did not influence the osteogenic differentiation of hBMMSCs in vivo.

Low concentration FFA suppressed bone loss in OVX mice and partially reversed bone loss in aged mice

To further demonstrate the clinical relevance of our results, whether FFA could suppress osteoporosis caused by ovariotomy and aging in mice was investigated. Forty 6-week-old female mice were divided into four groups of 10 mice: SHAM, SHAM+FFA, OVX, and OVX+FFA. No significant difference was found between SHAM mice with N.S. treatment and SHAM mice with FFA treatment in micro-CT and H\&E staining of the femurs (Fig. 4a), whereas FFA treatment partially blocked trabecular bone loss in the OVX mice. Moreover, in the OVX mice, there was a decrease in bone volume (Fig. 4b), trabecular number (Fig. 4b), trabecular thickness (Fig. 4b), and BMD (Fig. 4c) and an increase in trabecular separation (Fig. 4b), while treatment of FFA improved these parameters in the OVX mice, which were confirmed by bone histomorphometry analysis (Table 1). What is more, FFA treatment increased the bone formation marker P1NP (Fig. 4d) in serum of OVX mice.

Micro-CT and H\&E staining also showed that trabecular bone loss was partially reversed by FFA in aged mice (Fig. 4e). Specifically, compared with the aged mice treated with N.S., we observed an increase in bone volume (Fig. 4f), trabecular number (Fig. 4f), trabecular thickness (Fig. 4f), and BMD (Fig. 4g) and a reduction in trabecular separation (Fig. 4f) in the aged mice treated with FFA, which were confirmed by bone histomorphometry analysis (Table 2). What is more, FFA treatment increased the bone formation marker P1NP (Fig. 4h) in serum of aged mice.

Besides, FFA at the concentration used in the present study did not cause toxicity or inflammatory changes in the, kidney, liver, spleen, lung, and heart of the SHAM and aged mice (Additional file 3: Figure S3).

FFA at low concentrations promoted the osteogenic differentiation of hMSCs by inhibiting the NF- $\mathrm{KB}$ pathway.

Next, to explore the underlying mechanisms how FFA regulates osteogenic differentiation, cells treated with $50 \mu \mathrm{M}$ FFA in PM and OM were subjected to mRNA analysis for several key regulators of osteogenesis. To our surprise, in the pretest, we first found that $50 \mu \mathrm{M}$ FFA significantly attenuated the expression of the target genes of NF- $k B$, either in hBMMSCs cultured in PM or OM for 7 days (Fig. 5a). Based on this, we supposed the NF- $\mathrm{kB}$ signaling pathway was downregulated by FFA and that FFA at low concentrations promoted osteogenesis by inhibiting the NF- $\mathrm{BB}$ pathway. Therefore, we added the activators of NF- $\mathrm{kB}$ signaling pathway, TNF- $\alpha$, and LPS to OM with $50 \mu \mathrm{M}$ FFA. ALP staining and quantification showed that $50 \mu \mathrm{M}$ FFA no longer promoted osteogenesis of hBMMSCs in the presence of TNF- $\alpha$ or LPS (Fig. 5b, d). Meanwhile, the inhibitor of NF- $\mathrm{kB}$ signaling, Bay117082, enhanced the promoting effect of FFA on osteogenesis. The ARS staining and quantification on day 14 displayed results similar to those of ALP staining and quantification (Fig. 5c, e). Compared with OM with $50 \mu \mathrm{M}$ FFA, TNF- $\alpha$ and LPS significantly attenuated the expression level of RUNX2 (Fig. 5f) at day 7 and BGLAP (Fig. 5f) at day 14, whereas 
hASCs

PM

$\mathbf{a}$

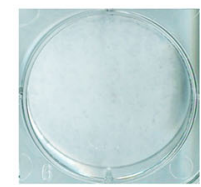

b

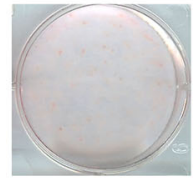

c

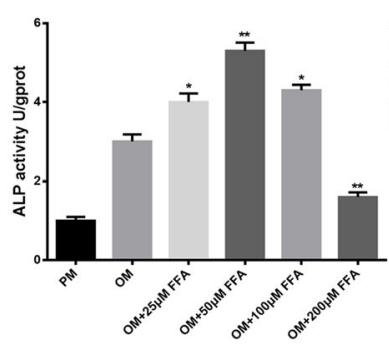

e

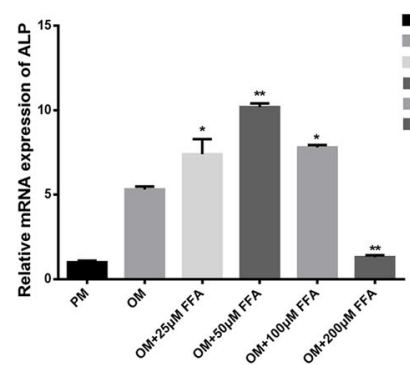

f

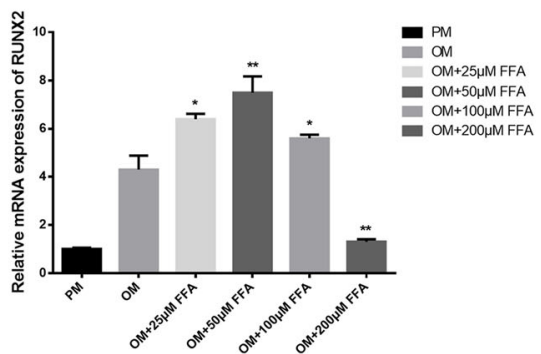

OM+FFA

$\begin{array}{llll}25 \mu \mathrm{M} & 50 \mu \mathrm{M} & 100 \mu \mathrm{M} & 200 \mu \mathrm{M}\end{array}$
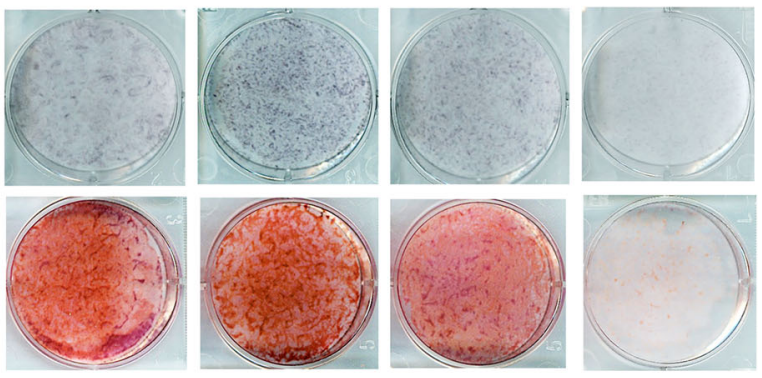

d

-

OM 25 M FFA

$O M+50 \mathrm{MMFA}$
$\mathrm{OM}+100 \mathrm{MMFFA}$

OMM+200MMFFA

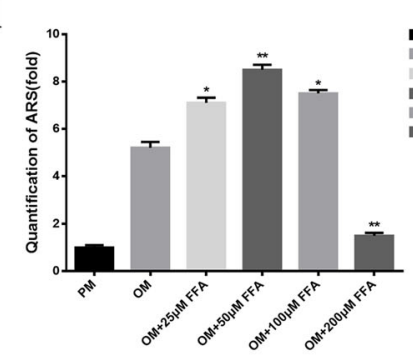

- $\begin{gathered}\mathrm{PM} \\ \mathrm{OM}\end{gathered}$

OM+25HM FFA

- OM+50MMFFA

OM+200MM FFA
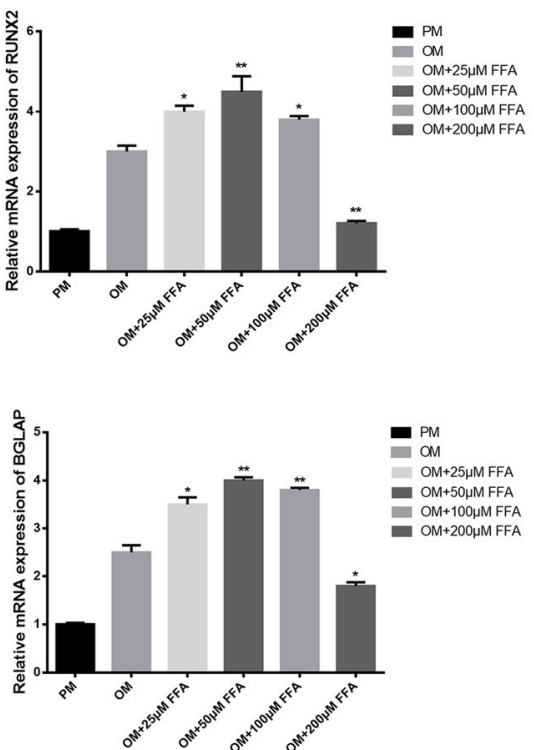

Fig. 2 FFA at different concentrations affected osteogenic differentiation of hASCs in vitro. a 25, 50, and $100 \mu \mathrm{M}$ FFA increased ALP activity in hASCs, whereas 200 MM FFA decreased ALP activity as shown by ALP staining. Human ASCs were cultured in PM, OM, or OM with FFA at 25 , 50,100 , or $200 \mu \mathrm{M}$ for 7 days. b FFA at 25, 50, and $100 \mu \mathrm{M}$ accelerated mineralization in hASCs, whereas $200 \mu \mathrm{M}$ FFA reduced mineralization as shown by ARS staining. Human ASCs were cultured in PM, OM, or OM with FFA at 25, 50,100, or $200 \mu \mathrm{M}$ for 14 days. c The result of quantification of ALP activity was consistent with the result of ALP staining. $\mathbf{d}$ The result of quantification of ARS was consistent with the result of ARS staining. e FFA at 25, 50, and $100 \mu \mathrm{M}$ promoted the expression of ALP and RUNX2 on day 7, whereas $200 \mu \mathrm{M}$ FFA inhibited the expression of the genes mentioned above, as determined by qRT-PCR. f FFA at 25,50 , and $100 \mu \mathrm{M}$ promoted the expression of RUNX2 and BGLAP on day 14 , whereas $200 \mu \mathrm{M}$ FFA inhibited the expression of the genes mentioned above, as determined by qRT-PCR in hASCs. All data are shown as the mean \pm SD, $n=3 .{ }^{*} p<0.05$ and ${ }^{* *} p<0.01$ compared with OM. FFA, flufenamic acid; hASCs, human adipose-derived stem cell; PM, proliferation media; OM, osteogenic media; ALP, alkaline phosphatase; ARS, alizarin red S; RUNX2, runt-related transcription factor 2; BGLAP, bone gammacarboxyglutamate protein; qRT-PCR, quantitative real-time reverse transcription PCR 

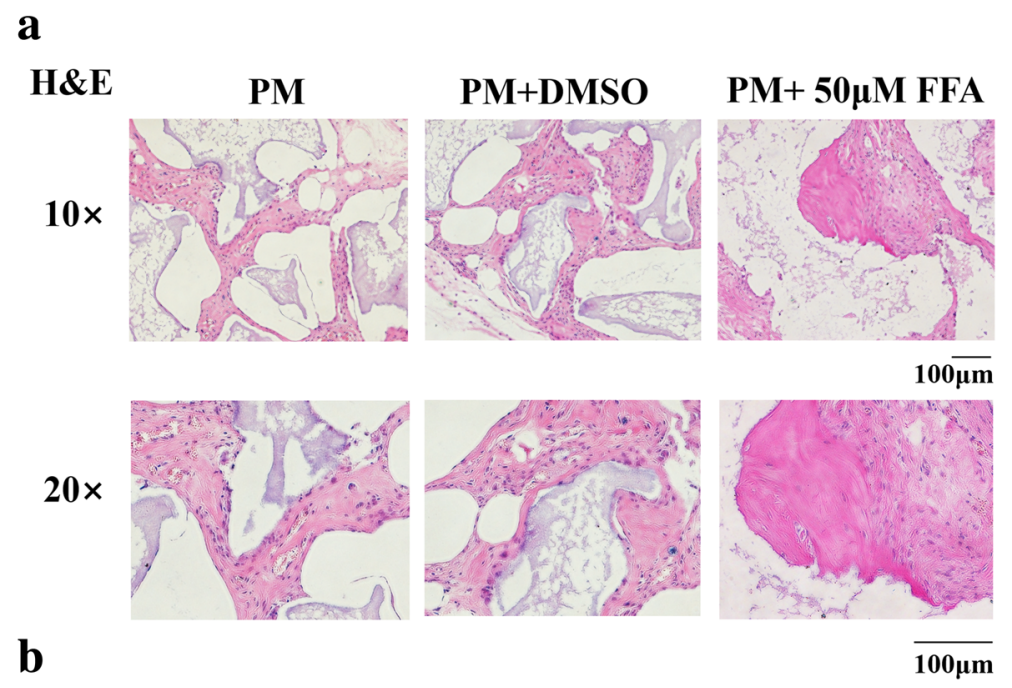

b

\section{Masson}
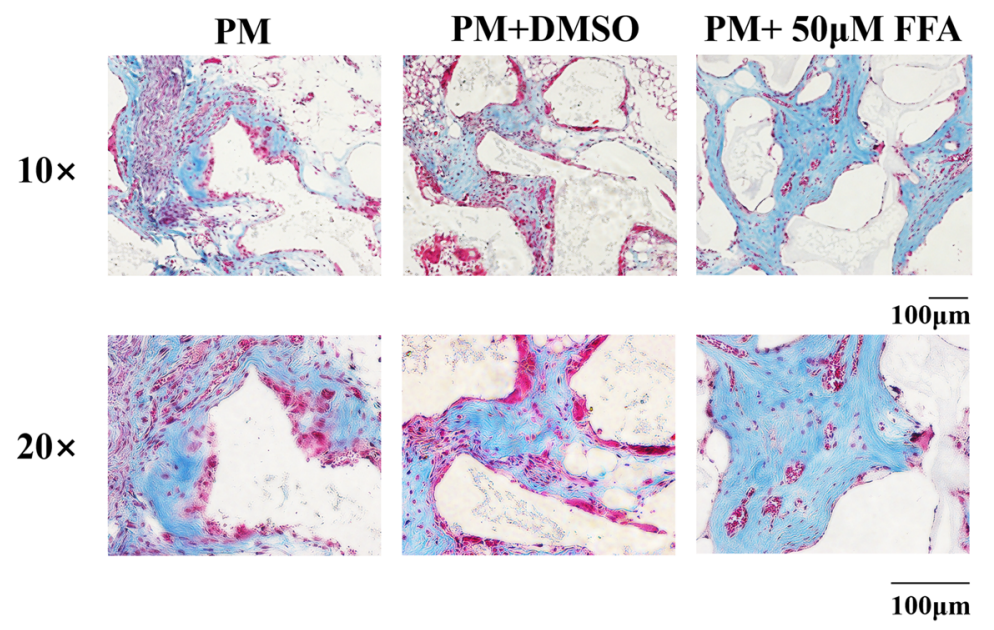

Fig. 3 FFA at $50 \mu \mathrm{M}$ promoted osteogenic differentiation of hBMMSCs in vivo. a H\&E staining of PM, PM+DMSO, and PM+FFA groups. b Masson's staining of PM, PM+DMSO, and PM+FFA groups. PM, proliferation media; FFA, flufenamic acid; hBMMSC, human bone marrow-derived mesenchymal stem cell; H\&E, hematoxylin and eosin

Bay117082 upregulated the expression. To further confirm the specific effect of FFA on NF- $\mathrm{kB}$ signaling pathway, we tested the effect of FFA on the key proteins of NF- $\mathrm{B}$ signaling pathway by western blot. Human BMMSCs were cultured in PM or PM with $50 \mu \mathrm{M}$ FFA for 7 days and then treated with TNF- $\alpha$ or LPS for 30 min. The results showed that in PM condition, TNF- $\alpha$ and LPS activated the phosphorylation of IKK and IKB $\alpha$ in $30 \mathrm{~min}$, while the total $\mathrm{I} \kappa \mathrm{B} \alpha$ was reduced. FFA treatment blocked the phosphorylation of IKK and IKB $\alpha$ as well as decreased total IKB $\alpha$ (Fig. 5g). Besides, FFA also blocked the phosphorylation of P65 activated by TNF- $\alpha$ and LPS and slightly decreased total P65 (Fig. 5 g). In addition, hBMMSCs were cultured in OM or OM with $50 \mu \mathrm{M}$ FFA for 7 days and then treated with TNF- $\alpha$ or LPS for $30 \mathrm{~min}$. In OM condition, FFA treatment not only had the same effect on the proteins tested in PM, but also increased the expression of RUNX2 remarkably (Fig. 5h).

\section{Discussion}

In this study, we found that FFA at low concentrations could promote osteogenic differentiation of hMSCs both in vitro and in vivo. We also observed that the effect declined as the concentration of FFA increased. In addition, the best concentration to promote osteogenesis was $50 \mu \mathrm{M}$. These results suggested that FFA at low concentrations might promote hMSC differentiation toward the osteoblastic lineage. Most importantly, we discovered that FFA not only promoted osteogenic differentiation effectively, but 


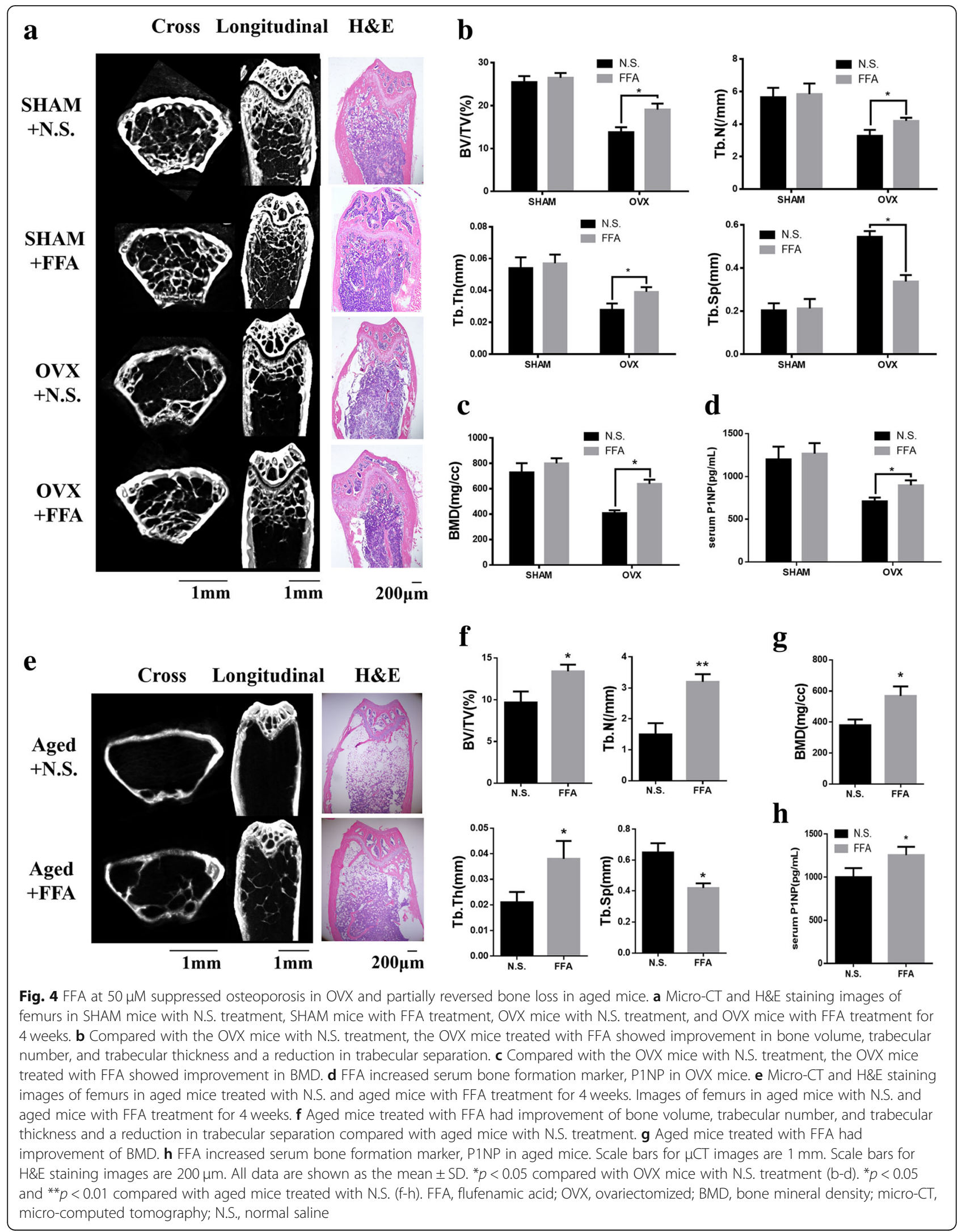


Table 1 Bone histomorphometry analysis of SHAM and OVX mice

\begin{tabular}{lllll}
\hline & SHAM+N.S. & SHAM+FFA & OVX+N.S. & OVX+FFA \\
\hline BV/TV $(\%)$ & $11.070 \pm 1.87$ & $12.532 \pm 1.92$ & $8.132 \pm 1.22$ & $9.370 \pm 1.10^{*}$ \\
Tb.Ar $(\%)$ & $5.315 \pm 0.42$ & $5.494 \pm 0.76$ & $3.570 \pm 0.52$ & $4.088 \pm 0.79$ \\
Tb.Th $(\mu \mathrm{m})$ & $11.874 \pm 1.35$ & $13.773 \pm 2.07^{*}$ & $7.385 \pm 0.86$ & $8.044 \pm 1.06$ \\
Tb.Sp $(\mu \mathrm{m})$ & $267.538 \pm 38.95$ & $255.379 \pm 27.66$ & $329.873 \pm 37.922$ & $295.552 \pm 26.816^{*}$ \\
Tb.N $(\# / \mathrm{mm})$ & $3.95 \pm 0.46$ & $4.23 \pm 0.59$ & $1.96 \pm 0.35$ & $2.31 \pm 0.29^{*}$ \\
MAR $(\mu \mathrm{m} /$ day) & $1.668 \pm 0.33$ & $1.709 \pm 0.29$ & $1.241 \pm 0.25$ & $1.533 \pm 0.31^{*}$ \\
BFR $(\mu \mathrm{m} /$ day) & $21.872 \pm 1.97$ & $20.511 \pm 2.85$ & $13.077 \pm 1.97$ & $14.472 \pm 3.69$ \\
Ob.S/BS $(\%)$ & $10.010 \pm 1.86$ & $11.677 \pm 1.98$ & $8.406 \pm 1.14$ & $9.002 \pm 1.37$ \\
N.Ob/T.Ar & $81.624 \pm 19.75$ & $92.331 \pm 10.948$ & $58.376 \pm 7.83$ & $67.679 \pm 9.03^{*}$ \\
N.Ob/B.Pm & $13.978 \pm 2.912$ & $13.470 \pm 4.011$ & $7.870 \pm 1.90$ & $9.010 \pm 1.65$
\end{tabular}

${ }^{*} p<0.05,{ }^{* *} p<0.01$, SHAM+FFA compared with SHAM+N.S., OVX+FFA compared with OVX + N.S

also suppressed bone loss in OVX mice and aged mice. Mechanistically, FFA promotes osteogenic differentiation by inhibiting the NF- $\mathrm{KB}$ signaling pathway. The present study provides valuable clues for a new use of FFA to treat bone metabolic diseases, such as osteoporosis.

The in vitro experiments in the present study revealed that FFA could affect the differentiation of hMSCs. Little evidence of the promoting effect of FFA at low concentrations on osteogenic differentiation of hMSCs was available in previous studies, except for a clinical case report which showed that chronic use of niflumic acid, a member of the fenamic acids, might lead to osteosclerosis [26]. This finding was strengthened by the promoting effect of $50 \mu \mathrm{M}$ FFA on the osteogenic differentiation of hMSCs in vivo and supported by the inhibiting effect of FFA on bone loss in OVX mice and aged mice. Taken together, this evidence indicated that FFA could play a role in promoting osteogenic differentiation of hMSCs and bone metabolism.

Table 2 Bone histomorphometry analysis of aged mice

\begin{tabular}{lll}
\hline & Aged+N.S. & Aged+FFA \\
\hline BV/TV $(\%)$ & $3.012 \pm 0.46$ & $4.035 \pm 0.39^{* * *}$ \\
Tb.Ar $(\%)$ & $2.615 \pm 0.32$ & $3.498 \pm 0.66^{* *}$ \\
Tb.Th $(\mu \mathrm{m})$ & $3.874 \pm 0.45$ & $4.373 \pm 0.57^{*}$ \\
Tb.Sp $(\mu \mathrm{m})$ & $589.783 \pm 66.01$ & $525.615 \pm 58.19^{*}$ \\
Tb.N $(\# / \mathrm{mm})$ & $1.05 \pm 0.26$ & $1.61 \pm 0.39^{* *}$ \\
MAR $(\mu \mathrm{m} /$ day $)$ & $0.893 \pm 0.13$ & $1.009 \pm 0.24$ \\
BFR $(\mu \mathrm{m} /$ day $)$ & $12.364 \pm 1.86$ & $13.196 \pm 2.35$ \\
Ob.S/BS $(\%)$ & $6.007 \pm 0.73$ & $6.462 \pm 1.04$ \\
N.Ob/T.Ar & $47.531 \pm 11.03$ & $55.198 \pm 9.038$ \\
N.Ob/B.Pm & $7.428 \pm 1.361$ & $8.598 \pm 1.023^{*}$ \\
\hline
\end{tabular}

${ }^{*} p<0.05,{ }^{* *} p<0.01,{ }^{* * *} p<0.001$
Several studies have focused on the effects of different NSAIDs on bones and osteocytes, including a series of biological behaviors such as adhesion, proliferation, and differentiation [27-32]. However, the effect differs with the change in drug and concentration. Perhaps, this is because the members of NSAIDs are quite different in their chemical structures and other properties, which may cause different effects, apart from their expected anti-inflammatory and analgesic behaviors. Currently, it is clear that aspirin can promote osteogenesis of MSCs and attenuate bone loss [10, 33-35]. However, the effective concentration is about $0.25-1.5 \mathrm{mmol}$ according to previous studies, which is far higher than the effective concentration of FFA. As the representative of fenamic acids, FFA is approved by US Food and Drug Administration (FDA) to treat several diseases in the clinic, such as rheumatic arthritis [36, 37]. FFA is inexpensive and can be obtained easily. Thus, our study provides clues for the application of FFA as a new, safe, and economical choice for osteoporosis treatment.

Our study revealed that FFA promoted osteogenesis of hMSCs by inhibition of the NF- $k B$ signaling pathway. NF- $\mathrm{kB}$ signaling pathways are associated with bone metabolism [14-19]. In addition, previous studies have shown that FFA can inhibit the NF- $\mathrm{kB}$ signaling pathway [38]. Our findings are in agreement with the previous studies. However, this study is the first to point out that FFA promotes osteogenesis through the NF- $\kappa B$ signaling pathway. Although the mechanism of action by which FFA promotes osteogenesis of hMSCs has not been completely elucidated, it is mediated, at least partly, by inhibition of NF-kB signaling. The proliferation curves of the cells showed that $200 \mu \mathrm{M}$ FFA inhibited the proliferation of hMSCs, which might partially account for the inhibiting effect of high concentration FFA on osteogenic differentiation of hMSCs. Besides, high dose of drugs may cause varied side effects, which means less 


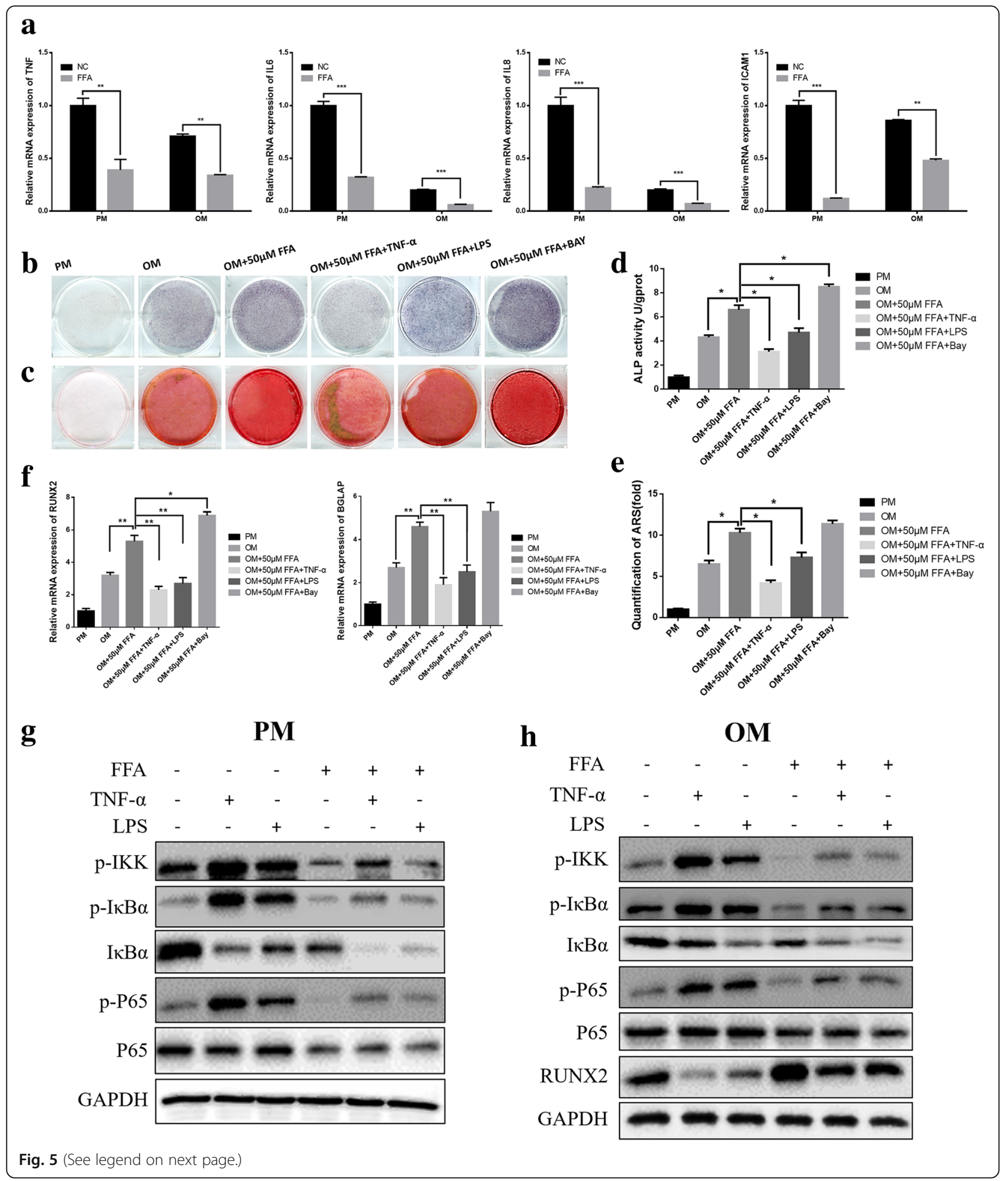


(See figure on previous page.)

Fig. 5 FFA in low concentrations promoted the osteogenic differentiation of hMSCs by inhibiting the NF-KB pathway. a FFA at $50 \mu M$ downregulated the expression of TNF, IL6, IL8, and ICAM1 both in PM and OM in hBMMSCs, as determined by qRT-PCR. b FFA at $50 \mu M$ could not increase ALP activity in hBMMSCs in the presence of TNF- $a$ or LPS. Yet FFA at $50 \mu \mathrm{M}$ increased ALP activity more in hBMMSCs in the presence of Bay 117082. Human BMMSCs were treated with PM, OM, OM with FFA at $50 \mu \mathrm{M}$, or OM with FFA at $50 \mu \mathrm{M}$ and TNF-a or LPS or Bay 117082 for 7 days before ALP staining. c FFA at $50 \mu \mathrm{M}$ no longer accelerated mineralization in hBMMSCs in the presence of TNF-a or LPS. Yet FFA at $50 \mu M$ accelerated mineralization more in hBMMSCs in the presence of Bay117082. Cells were treated with PM, OM, OM with FFA at $50 \mu M$, or OM with FFA at $50 \mu \mathrm{M}$ and TNF-a or LPS or Bay 117082 for 14 days, and calcium deposition was then tested using ARS staining. d The result of quantification of ALP activity was consistent with the result of ALP staining. $\mathbf{e}$ The result of quantification of ARS was consistent with the result of ARS staining. f TNF- $a$ and LPS downregulated the expression of RUNX2 and BGLAP in hBMMSCs, which had been increased by $50 \mu M$ FFA, as determined by qRT-PCR. Bay117082 further upregulated the expression of RUNX2 and BGLAP in hBMMSCs, which had been increased by $50 \mu M$ FFA, as determined by qRT-PCR. $\mathbf{g}$ Western blot of protein expression of p-IKK, p-IkBa, IkBa, p-P65, P65, and the internal control GAPDH. Human BMMSCs were cultured in PM or PM with FFA for 7 days before treated with TNF- $a$ or LPS for $30 \mathrm{~min}$. $\mathbf{h}$ Western blot of protein expression of $p$ IKK, p-IKBa, IKBa, p-P65, P65, RUNX2, and the internal control GAPDH. Human BMMSCs were cultured in OM or OM with FFA for 7 days before treated with TNF-a or LPS for 30 min. All data are shown as the mean \pm SD, $n=3 .{ }^{*} p<0.05,{ }^{* *} p<0.01$, and ${ }^{* * *} p<0.001$. FFA, flufenamic acid; hBMMSC, human bone marrow-derived mesenchymal stem cell; PM, proliferation media; OM, osteogenic media; ALP, alkaline phosphatase; ARS, alizarin red S; RUNX2, runt-related transcription factor 2; BGLAP, bone gamma-carboxyglutamate protein; qRT-PCR, quantitative real-time reverse transcription PCR; hMSC, human mesenchymal stem cell; NF-KB, nuclear factor kappa B; TNF-a, tumor necrosis factor alpha

value in clinic. Thus, we did not deeply study the mechanism how FFA at high concentration inhibited osteogenic differentiation of hMSCs.

However, our study still has several limitations. We only evaluated the signaling pathways by which FFA at low concentrations promoted osteogenesis of hMSCs and partially explained why high concentration FFA inhibited osteogenesis of hMSCs. The complete reason remains unclear. Besides, according to our results, it was clear that the best concentration to promote osteogenesis was between 25 and $100 \mu \mathrm{M}$, and among the chosen concentrations in the present study, $50 \mu \mathrm{M}$ FFA had the best promoting effect. However, some concentrations between 25 and $100 \mu \mathrm{M}$ might have better effect than $50 \mu \mathrm{M}$ and further studies are needed to determine the optimal concentration. Moreover, to promote its clinical transformation, further evaluation of the specific treatment concentration in humans is required.

Overall, our results showed that FFA at low concentrations could promote osteogenic differentiation of hMSCs, both in vitro and in vivo, by inhibition of NF- $\mathrm{kB}$ signaling. It could also suppress bone loss caused by ovariotomy and aging in mice, suggesting that FFA has a potential therapeutic value to treat osteoporosis.

\section{Conclusion}

FFA in low concentration promotes osteogenic differentiation of hMSCs in vitro, and the effect declines with increasing concentration. The best concentration for osteogenesis promoting is $50 \mu \mathrm{M}$. Fifty micromolar FFA can also promote osteogenic differentiation of hMSCs in vivo. Besides, $50 \mu \mathrm{M}$ FFA suppress bone loss in OVX mice and aged mice. Mechanistically, FFA promotes osteogenesis through inhibiting NF- $\mathrm{B}$ signaling pathway. Collectively, our study suggested that FFA could be used in bone tissue engineering or osteoporosis by promoting osteogenic differentiation of human MSCs.

\section{Additional files}

\begin{abstract}
Additional file 1: Figure S1. DMSO (1\%) did not affect the osteogenic differentiation of hMSCs. a 1\%o DMSO did not affect ALP activity in hBMMSCs as tested by ALP staining. b 1\%o DMSO did not affect ALP activity in hASCs as tested by ALP staining. ALP, alkaline phosphatase; hMSC, human mesenchymal stem cell; hBMMSC, human bone marrowderived mesenchymal stem cell; hASC, human adipose derived stem cell. (TIF 744 kb)
\end{abstract}

Additional file 2: Figure S2. High concentration FFA inhibited proliferation of hMSCs. a 200 MM FFA inhibited proliferation of hBMMSCs whereas 25, 50, and $100 \mu \mathrm{M}$ FFA caused no significant differences in the proliferative capacities of the cells compared with no FFA treatment during day 1 (1) to day 7 (7), as shown by the growth curve of cells. b $200 \mu \mathrm{M}$ FFA inhibited proliferation of hASCs whereas 25,50, and $100 \mu \mathrm{M}$ FFA caused no significant differences in the proliferative capacities of the cells compared with no FFA treatment during day 1 (1) to day 7 (7), as shown by the growth curve of cells. FFA, flufenamic acid; hMSC, human mesenchymal stem cell; hASC, human adipose derived stem cell; hBMMSC, human bone marrow-derived mesenchymal stem cell. (TIF 373 kb)

Additional file 3: Figure S3. FFA caused no toxicity or inflammation in the viscera of mice. Intraperitoneal injection of FFA for 1 month did not cause toxicity or inflammation changes in the kidney, liver, spleen, lung, or heart of SHAM and aged mice. Scale bar $=500 \mu \mathrm{m}$. FFA, flufenamic acid. (TIF 11408 kb)

\section{Abbreviations}

ALP: Alkaline phosphatase; ARS: Alizarin red S; BGLAP: Bone gammacarboxyglutamic acid-containing protein; BMD: Bone mineral density; BMMSCs: Bone marrow mesenchymal stem cells; BV/TV: Trabecular bone volume/tissue volume; DMSO: Dimethyl sulfoxide; ELISA: Enzyme-linked immunosorbent assay; FFA: Flufenamic acid; GAPDH: Glyceraldehyde 3phosphate dehydrogenase; H\&E: Hematoxylin and eosin; hASCs: Human adipose-derived stem cells; ICAM1: Intercellular adhesion molecule 1; IL: Interleukin; LPS: Lipopolysaccharide; Micro-CT: Micro-computed tomography; MSCs: Mesenchymal stem cells; NF-KB: Nuclear factor-KB; NSAIDs: Non-steroidal anti-inflammatory drugs; OCN: Osteocalcin; 
OM: Osteogenic medium; P1NP: Procollagen I N-terminal propeptide; PM: Proliferation medium; qRT-PCR: Quantitative reverse transcription PCR; RUNX2: Runt-related transcription factor 2; Tb.N: Trabecular number; Tb.Sp: Trabecular spacing; Tb.Th: Trabecular thickness; TNF: Tumor necrosis factor

\section{Acknowledgements}

The authors are grateful to Dr. Wensi Wang for the help in animal operations.

\section{Authors' contributions}

$\mathrm{XL}$ was responsible for the conception and design, collection and/or assembly of data, data analyses and interpretation, and manuscript writing. $\mathrm{ZL}, \mathrm{HL}$, and $\mathrm{YZ}$ were responsible for the collection and/or assembly of data and data analyses and interpretation in the animal experiments. DX, SW, RG and WW were responsible for the collection and/or assembly of data and data analyses in the molecular biology experiments. PZ and YL were responsible for the conception and design, financial support, and manuscript writing. YZ was responsible for the conception and design and manuscript writing. All authors read and approved the final manuscript.

\section{Funding}

This study was supported by grants from the National Key Research and Development Program of China (No. 2016 YFC1102900 to YL), the National Natural Science Foundation of China (No. 81771039 to YL), Peking University Medicine Seed Fund for Interdisciplinary Research (No. BMU2018ME005 to YL), and the Beijing Nova Program (No. Z181100006218037 to PZ).

\section{Availability of data and materials}

The authors confirm that all data underlying the findings are fully available.

\section{Ethics approval and consent to participate}

This study was carried out in strict accordance with the recommendations of the Guide for the Care and Use of Laboratory Animals of the National Institutes of Health. The protocol was approved by the Institutional Animal Care and Use Committee of the Peking University Health Science Center (approval no. LA2014233). All surgeries were performed under anesthesia, and all efforts were made to minimize animal suffering.

\section{Consent for publication}

Not applicable.

\section{Competing interests}

The authors declare that they have no competing interests.

\section{Author details}

${ }^{1}$ Department of Prosthodontics, Peking University School and Hospital of Stomatology, National Laboratory for Digital and Material Technology of Stomatology, Beijing Key Laboratory of Digital Stomatology, National Clinical Research Center for Oral Diseases, 22 Zhongguancun South Avenue, Beijing 100081, People's Republic of China. ${ }^{2}$ Department of Materials Science and Engineering, College of Engineering, Peking University, Beijing 100871, People's Republic of China. ${ }^{3}$ Department of Implantology II, The Affiliated Stomatological Hospital of Fujian Medical University, Fuzhou 350001, Fujian, People's Republic of China.

Received: 8 April 2019 Revised: 9 June 2019 Accepted: 30 June 2019 Published online: 19 July 2019

\section{References}

1. Hennemann A. Osteoporosis: prevention, diagnosis and therapy. Med Monatsschr Pharm. 2002;25(5):164-7.

2. Turner RT, Riggs BL, Spelsberg TC. Skeletal effects of estrogen. Endocr Rev. 1994;15(3):275-300.

3. Hess R, Pino AM, Rios S, Fernandez M, Rodriguez JP. High affinity leptin receptors are present in human mesenchymal stem cells (MSCs) derived from control and osteoporotic donors. J Cell Biochem. 2005;94(1):50-7.

4. Dalle Carbonare L, Valenti MT, Zanatta M, Donatelli L, Lo Cascio V. Circulating mesenchymal stem cells with abnormal osteogenic differentiation in patients with osteoporosis. Arthritis Rheum. 2009;60(11): 3356-65.
5. Post S, Abdallah BM, Bentzon JF, Kassem M. Demonstration of the presence of independent pre-osteoblastic and pre-adipocytic cell populations in bone marrow-derived mesenchymal stem cells. Bone. 2008;43(1):32-9.

6. Vane JR. Inhibition of prostaglandin synthesis as a mechanism of action for aspirin-like drugs. Nat New Biol. 1971;231(25):232-5.

7. Vane JR. The mode of action of aspirin and similar compounds. J Allergy Clin Immunol. 1976;58(6):691-712.

8. Moncada S, Ferreira SH, Vane JR. Prostaglandins, aspirin-like drugs and the oedema of inflammation. Nature. 1973;246(5430):217-9.

9. Sriram D, Yogeeswari P. Medicinal chemistry. 2nd ed. New Delhi: Pearson Education India; 2010. p. 235.

10. Liu H, Li W, Liu Y, Zhang X, Zhou Y. Co-administration of aspirin and allogeneic adipose-derived stromal cells attenuates bone loss in ovariectomized rats through the anti-inflammatory and chemotactic abilities of aspirin. Stem Cell Res Ther. 2015:6:200

11. Hadjicharalambous C, Alexaki Vl, Alpantaki K, Chatzinikolaidou M. Effects of NSAIDs on the osteogenic differentiation of human adipose tissue-derived stromal cells. J Pharm Pharmacol. 2016;68(11):1403-8.

12. Lawrence T, Bebien M, Liu GY, Nizet V, Karin M. IKKalpha limits macrophage NF-kappaB activation and contributes to the resolution of inflammation. Nature. 2005;434(7037):1138-43.

13. Rius J, Guma M, Schachtrup C, Akassoglou K, Zinkernagel AS, Nizet V, et al. NF-kappaB links innate immunity to the hypoxic response through transcriptional regulation of HIF-1alpha. Nature. 2008;453(7196):807-11.

14. Chang J, Liu F, Lee M, Wu B, Ting K, Zara JN, et al. NF-kappaB inhibits osteogenic differentiation of mesenchymal stem cells by promoting betacatenin degradation. Proc Natl Acad Sci U S A. 2013;110(23):9469-74.

15. Chang J, Wang Z, Tang E, Fan Z, McCauley L, Franceschi R, et al. Inhibition of osteoblastic bone formation by nuclear factor-kappaB. Nat Med. 2009: 15(6):682-9.

16. Chen Q, Liu K, Robinson AR, Clauson CL, Blair HC, Robbins PD, et al. DNA damage drives accelerated bone aging via an NF-kappaB-dependent mechanism. J Bone Miner Res. 2013;28(5):1214-28.

17. Novack DV. Role of NF-kappaB in the skeleton. Cell Res. 2011;21(1):169-82.

18. Li Y, Li A, Strait K, Zhang H, Nanes MS, Weitzmann MN. Endogenous TNFalpha lowers maximum peak bone mass and inhibits osteoblastic Smad activation through NF-kappaB. J Bone Miner Res. 2007;22(5):646-55.

19. Tarapore RS, Lim J, Tian C, Pacios S, Xiao W, Reid D, et al. NF-kappaB has a direct role in inhibiting Bmp- and Wnt-induced matrix protein expression. J Bone Miner Res. 2016:31(1):52-64.

20. Zhang P, Liu Y, Jin C, Zhang M, Tang F, Zhou Y. Histone acetyltransferase GCN5 regulates osteogenic differentiation of mesenchymal stem cells by inhibiting NF-kappaB. J Bone Miner Res. 2016;31(2):391-402.

21. Huang J, Hsu YH, Mo C, Abreu E, Kiel DP, Bonewald LF, et al. METTL21C is a potential pleiotropic gene for osteoporosis and sarcopenia acting through the modulation of the NF-kappaB signaling pathway. J Bone Miner Res. 2014;29(7):1531-40.

22. Ducy P, Desbois C, Boyce B, Pinero G, Story B, Dunstan C, et al. Increased bone formation in osteocalcin-deficient mice. Nature. 1996:382(6590):44852

23. Jin C, Zhang P, Zhang M, Zhang X, LV L, Liu H, et al. Inhibition of SLC7A11 by sulfasalazine enhances osteogenic differentiation of mesenchymal stem cells by modulating BMP2/4 expression and suppresses bone loss in ovariectomized mice. J Bone Miner Res. 2017;32(3):508-21.

24. Lv L, Ge W, Liu Y, Lai G, Liu H, Li W, et al. Lysine-specific demethylase 1 inhibitor rescues the osteogenic ability of mesenchymal stem cells under osteoporotic conditions by modulating H3K4 methylation. Bone Res. 2016;4: 16037.

25. Kua HY, Liu H, Leong WF, Li L, Jia D, Ma G, et al. c-Abl promotes osteoblast expansion by differentially regulating canonical and non-canonical BMP pathways and p16INK4a expression. Nat Cell Biol. 2012;14(7):727-37.

26. Meunier PJ, Courpron P, Smoller JS, Briancon D. Niflumic acid-induced skeletal fluorosis: iatrogenic disease or therapeutic perspective forosteoporosis? Clin Orthop Relat Res. 1980;148:304-9.

27. Chang JK, Li CJ, Liao HJ, Wang CK, Wang GJ, Ho ML. Anti-inflammatory drugs suppress proliferation and induce apoptosis through altering expressions of cell cycle regulators and pro-apoptotic factors in cultured human osteoblasts. Toxicology. 2009;258(2-3):148-56.

28. Chang JK, Li CJ, Wu SC, Yeh CH, Chen CH, Fu YC, et al. Effects of antiinflammatory drugs on proliferation, cytotoxicity and osteogenesis in bone marrow mesenchymal stem cells. Biochem Pharmacol. 2007;74(9):1371-82. 
29. De Luna-Bertos E, Ramos-Torrecillas J, Manzano-Moreno FJ, Garcia-Martinez $\mathrm{O}$, Ruiz C. Effects on growth of human osteoblast-like cells of three nonsteroidal anti-inflammatory drugs: metamizole, dexketoprofen, and ketorolac. Biol Res Nurs. 2015;17(1):62-7.

30. Diaz-Rodriguez L, Garcia-Martinez O, Morales MA, Rodriguez-Perez L, RubioRuiz B, Ruiz C. Effects of indomethacin, nimesulide, and diclofenac on human MG-63 osteosarcoma cell line. Biol Res Nurs. 2012;14(1):98-107.

31. Evans $C E$, Butcher $C$. The influence on human osteoblasts in vitro of nonsteroidal anti-inflammatory drugs which act on different cyclooxygenase enzymes. J Bone Joint Surg Br. 2004;86(3):444-9.

32. Garcia-Martinez O, Diaz-Rodriguez L, Rodriguez-Perez L, De Luna-Bertos E, Reyes Botella C, Ruiz CC. Effect of acetaminophen, ibuprofen and methylprednisolone on different parameters of human osteoblast-like cells. Arch Oral Biol. 2011;56(4):317-23.

33. Liu Y, Wang L, Kikuiri T, Akiyama K, Chen C, Xu X, et al. Mesenchymal stem cell-based tissue regeneration is governed by recipient $T$ lymphocytes via IFN-gamma and TNF-alpha. Nat Med. 2011;17(12):1594-601.

34. Lin S, Lee WYW, Huang M, Fu Z, Liang Y, Wu H, et al. Aspirin prevents bone loss with little mechanical improvement in high-fat-fed ovariectomized rats. Eur J Pharmacol. 2016;791:331-8.

35. Cao Y, Xiong J, Mei S, Wang F, Zhao Z, Wang S, et al. Aspirin promotes bone marrow mesenchymal stem cell-based calvarial bone regeneration in mini swine. Stem Cell Res Ther. 2015;6:210.

36. Barnardo DE, Currey HL, Mason RM, Fox WR, Weatherall M. Mefenamic acid and flufenamic acid compared with aspirin and phenylbutazone in rheumatoid arthritis. Br Med J. 1966;2(5509):342-3.

37. Rajan KT, Hill AG, Barr A, Whitwell E. Flufenamic acid in rheumatoid arthritis. Ann Rheum Dis. 1967;26(1):43-6.

38. Pongkorpsakol P, Satitsri S, Wongkrasant P, Chittavanich P, Kittayaruksakul S, Srimanote $P$, et al. Flufenamic acid protects against intestinal fluid secretion and barrier leakage in a mouse model of Vibrio cholerae infection through NF-kappaB inhibition and AMPK activation. Eur J Pharmacol. 2017;798:94104.

\section{Publisher's Note}

Springer Nature remains neutral with regard to jurisdictional claims in published maps and institutional affiliations.

Ready to submit your research? Choose BMC and benefit from:

- fast, convenient online submission

- thorough peer review by experienced researchers in your field

- rapid publication on acceptance

- support for research data, including large and complex data types

- gold Open Access which fosters wider collaboration and increased citations

- maximum visibility for your research: over $100 \mathrm{M}$ website views per year

At $\mathrm{BMC}$, research is always in progress.

Learn more biomedcentral.com/submissions 